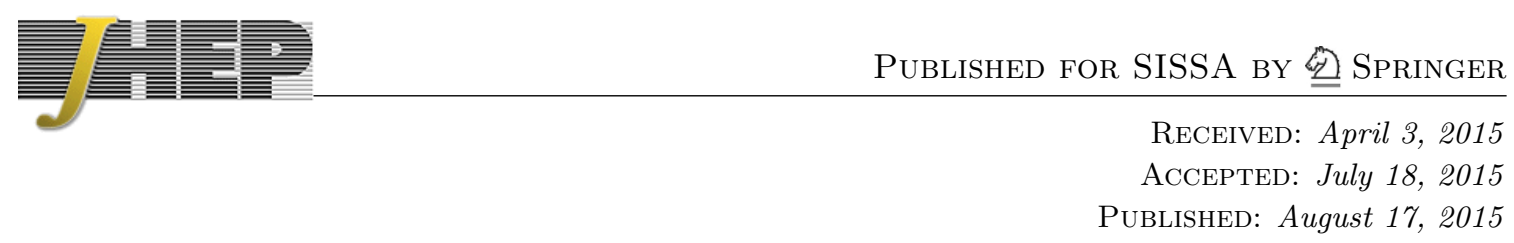

\title{
A couplet from flavored dark matter
}

\author{
Prateek Agrawal, $^{a}$ Zackaria Chacko, $^{b}$ Can Kilic ${ }^{c}$ and Christopher B. Verhaaren ${ }^{b}$ \\ ${ }^{a}$ Fermilab, \\ P.O. Box 500, Batavia, IL, 60510 U.S.A. \\ ${ }^{b}$ Maryland Center for Fundamental Physics, Department of Physics, University of Maryland, \\ College Park, MD, 20742-4111 U.S.A. \\ ${ }^{c}$ Theory Group, Department of Physics and Texas Cosmology Center, \\ The University of Texas at Austin, 2515 Speedway Stop C1608, Austin, TX, 78712-1197 U.S.A. \\ E-mail: prateek@fnal.gov, zchacko@umd.edu, kilic@physics.utexas.edu, \\ cver@umd.edu
}

ABSTRACT: We show that a couplet, a pair of closely spaced photon lines, in the X-ray spectrum is a distinctive feature of lepton flavored dark matter models for which the mass spectrum is dictated by Minimal Flavor Violation. In such a scenario, mass splittings between different dark matter flavors are determined by Standard Model Yukawa couplings and can naturally be small, allowing all three flavors to be long-lived and contribute to the observed abundance. Then, in the presence of a tiny source of flavor violation, heavier dark matter flavors can decay via a dipole transition on cosmological timescales, giving rise to three photon lines. Two of these lines are closely spaced, and constitute the couplet. Provided the flavor violation is sufficiently small, the ratios of the line energies are determined in terms of the charged lepton masses, and constitute a prediction of this framework. For dark matter masses of order the weak scale, the couplet lies in the $\mathrm{keV}-\mathrm{MeV}$ region, with a much weaker line in the $\mathrm{eV}-\mathrm{keV}$ region. This scenario constitutes a potential explanation for the recent claim of the observation of a $3.5 \mathrm{keV}$ line. The next generation of X-ray telescopes may have the necessary resolution to resolve the double line structure of such a couplet.

Keywords: Beyond Standard Model, Cosmology of Theories beyond the SM, Global Symmetries

ARXIV EPRINT: 1503.03057 


\section{Contents}

1 Introduction 1

2 The framework 3

3 A benchmark model $\quad 5$

3.1 Mass splittings 5

$\begin{array}{lll}3.2 & \text { Relic abundance } & 7\end{array}$

3.3 Direct detection $\quad 9$

3.4 Indirect detection 9

$\begin{array}{ll}3.5 & \text { The couplet } \\ 3.6 & 10\end{array}$

3.6 The $3.5 \mathrm{keV}$ line 11

4 Conclusions $\quad 13$

\section{Introduction}

Increasingly precise cosmological measurements indicate that about $80 \%$ of the matter density of the universe is composed of particles that are non-baryonic, and neutral under both color and electromagnetic interactions. However, the precise nature of this dark matter (DM) remains a mystery. One theoretically appealing possibility is that DM is composed of Weakly Interacting Massive Particles (WIMPs), particles with mass of order the weak scale that have interactions of weak scale strength with the standard model (SM) fields. This scenario is compelling because, provided the WIMPs were in thermal equilibrium with the SM at early times, just enough of them survive today as thermal relics to account for the observed DM density.

While the WIMP framework requires that DM have interactions of weak scale strength with the SM fields, efforts to produce it at high energy colliders have, so far, proven fruitless. Likewise, efforts to directly detect DM in the laboratory through its scattering off nucleons, in spite of the increased sensitivity of current experiments, have also been unsuccessful. There are some tentative hints from indirect detection of DM annihilation to SM today, but there is no conclusive signal. In the wake of these null results, DM scenarios that retain the cosmological success of the WIMP framework while satisfying the current experimental bounds have become increasingly compelling.

The matter fields of the $\mathrm{SM}\left(Q, U^{c}, D^{c}, L\right.$ and $\left.E^{c}\right)$ are known to come in three copies, or flavors. Different flavors carry the same charges under the SM gauge groups, but have different couplings to the Higgs, and so differ in their masses. One interesting possibility, which has been receiving increased attention, is that DM, like the SM matter fields, also comes in three flavors [1-11] or has flavor-specific couplings to the SM [12-15]. Specific 
DM candidates of this type include sneutrino DM in supersymmetric extensions of the SM [16], and Kaluza-Klein neutrino DM in theories with a universal extra dimension.

In [2], the simplest theories of flavored DM (FDM) were classified, and labelled as lepton flavored, quark flavored or internal flavored, based on the form of the interactions of the DM candidate with the SM fields. Models in which the DM has tree level interactions with the SM leptons but not with the quarks - as in lepton FDM — can naturally account for the observed abundance of DM while remaining consistent with all experimental bounds [17-19]. The reason is that strong production at a hadron collider or scattering off a nucleus rely on DM-quark interactions, which are loop suppressed in this scenario. In addition, because the average number of photons generated in DM annihilation to hadrons is much larger than in the case of DM annihilation to leptons, the limits from indirect DM searches using gamma rays are also weaker. Some other indirect signals of DM annihilation, such as the positron flux, are enhanced for lepton FDM, but regions of parameter space for which the DM is a thermal relic remain viable.

In general the couplings of lepton FDM violate the flavor symmetries of the SM. In order to avoid conflict with the very stringent bounds on flavor violating processes such as $\mu \rightarrow e \gamma$, while giving rise to an annihilation cross section of weak scale strength, the couplings of DM to the SM leptons must be aligned with the SM Yukawa interactions. In theories where the flavor structure is consistent with Minimal Flavor Violation (MFV), so that the only sources of flavor violation are associated with the SM Yukawa couplings, this condition is automatically satisfied. Then each DM flavor is associated with a corresponding lepton flavor. It is this class of theories that we shall focus on.

In realizations of FDM that respect MFV, the mass splitting between a pair of different DM flavors is dictated by the corresponding SM Yukawa couplings. In simple models, this splitting is proportional to the difference in the squares of the Yukawa couplings, so that for Dirac fermions we obtain,

$$
m_{\chi, i}-m_{\chi, j} \simeq \eta\left(y_{i}^{2}-y_{j}^{2}\right)
$$

In this expression the index $i=1,2,3$ runs over the three lepton flavors $e, \mu, \tau$. While $m_{\chi, i}$ represents the mass of the $i^{\text {th }}$ DM flavor, and $y_{i}$ represents the Yukawa coupling of the $i^{\text {th }}$ lepton flavor. The constant $\eta$ has the dimensions of mass and depends on the dynamics which UV completes flavor at some high scale $\Lambda$. If threshold effects at this scale generate mass splittings at tree level, $\eta$ can naturally be of order $m_{\chi}$, where $m_{\chi}$ is the average DM mass. In this case the largest splitting, that between the $e$ and $\tau$ flavors, is expected to be in the $\mathrm{MeV}-\mathrm{GeV}$ range for weak scale DM. If tree-level contributions at the threshold are absent, the leading effects are then loop suppressed. The largest splitting is then much smaller, in the keV-MeV range.

Since the Yukawa couplings are small, the splittings between the different DM flavors are suppressed relative to the mass of each flavor. If the splittings are smaller than the electron mass, the dominant flavor-conserving decay mode,

$$
\chi_{i} \rightarrow \chi_{j}+\nu_{i}+\bar{\nu}_{j}
$$


is slow on cosmological timescales, so that the lifetimes of the heavier flavors are much longer than the age of the universe. Then all three flavors are expected to contribute to the observed DM abundance.

Now, suppose a tiny additional source of explicit flavor breaking is present in the theory, so that the flavor violating decays,

$$
\chi_{i} \rightarrow \chi_{j}+\gamma
$$

can occur on cosmological timescales and dominate over the flavor-conserving decay. The monochromatic photons produced in such decays then constitute a striking signal of DM. Provided this new source of flavor violation is too small to contribute significantly to the splittings between the different DM flavors, the frequencies of the resulting gamma ray lines depend on the SM Yukawa couplings as in eq. (1.1). The constant of proportionality in eq. (1.1) cancels out when ratios of frequencies are considered. For example, if the $\tau$ flavor of DM is the heaviest and $\chi_{e}$ the lightest, we have,

$$
\frac{\omega\left(\chi_{\tau} \rightarrow \chi_{\mu}\right)}{\omega\left(\chi_{\tau} \rightarrow \chi_{e}\right)}=\frac{m_{\tau}^{2}-m_{\mu}^{2}}{m_{\tau}^{2}-m_{e}^{2}} \approx 1-\frac{m_{\mu}^{2}}{m_{\tau}^{2}}
$$

and,

$$
\frac{\omega\left(\chi_{\mu} \rightarrow \chi_{e}\right)}{\omega\left(\chi_{\tau} \rightarrow \chi_{e}\right)}=\frac{m_{\mu}^{2}-m_{e}^{2}}{m_{\tau}^{2}-m_{e}^{2}} \approx \frac{m_{\mu}^{2}}{m_{\tau}^{2}}=3.5 \times 10^{-3} .
$$

We see that this scenario predicts a pair of very closely spaced lines in the keV-MeV region corresponding to the $\chi_{\tau} \rightarrow \chi_{e}$ and $\chi_{\tau} \rightarrow \chi_{\mu}$ transitions (the "couplet"), as well as an isolated line in the $\mathrm{eV}-\mathrm{keV}$ region. Remarkably, we see that in this limit of negligible flavor violation, the ratios of these lines frequencies are a prediction of this scenario.

The fact that decaying DM particles will, in general, be in motion, leads to some broadening of these lines. The size of this effect scales with the velocity of DM. For typical astrophysical sources, the DM velocity $v$ ranges from $(1-3) \times 10^{-3}$, and a line of frequency $\omega$ is smeared by order $v \omega$. Comparing to eq. (1.5), we see that whether the double line feature gets washed out by this effect depends on the astrophysical source. While this splitting is not currently measurable, it is within the design resolution of upcoming experiments such as ASTRO-H [20, 21].

In the next section we review the MFV framework for models of lepton flavored DM. In section 3 we choose a simple benchmark to illustrate the phenomenology of this scenario, focusing on constraints from various DM experiments and potential signals. We conclude in section 4 .

\section{The framework}

In this section we study the restrictions that MFV places on the parameters of theories of lepton FDM. The SM has an approximate $\mathrm{U}(3)^{5}$ flavor symmetry that acts on the three generations of fermions $Q, U^{c}, D^{c}, L$ and $E^{c}$. This symmetry is explicitly broken by the SM Yukawa couplings. In extensions of the SM that respect MFV, any new parameter 
that violates the SM flavor symmetries must be aligned with the SM Yukawa couplings. Specifically, the Yukawa couplings of the SM are promoted to spurions that transform under the $\mathrm{U}(3)^{5}$ flavor symmetry. Any new interactions are then required to be invariant under this spurious symmetry.

In the lepton sector of the SM there is an approximate $\mathrm{U}(3)_{L} \times \mathrm{U}(3)_{E}$ flavor symmetry that acts on the left-handed $\mathrm{SU}(2)$ doublets $L^{A}$ and $\mathrm{SU}(2)$ singlets $E_{i}^{c}$. We denote $\mathrm{U}(3)_{L}$ indices by capital Latin letters and $\mathrm{U}(3)_{E}$ indices by lowercase Latin letters. This symmetry is violated by the SM Yukawa interactions,

$$
\mathcal{L}_{\mathrm{Y}}=Y_{A}{ }^{i} L^{A} H^{\dagger} E_{i}^{c}+\text { h.c. }
$$

We can, however, make the Yukawa interactions formally invariant under the flavor symmetry by promoting the matrix $Y_{A}{ }^{i}$ to be a spurion that transforms as $(\mathbf{3}, \overline{\mathbf{3}})$ under $\mathrm{SU}(3)_{L} \times \mathrm{SU}(3)_{E}$ subgroup of $\mathrm{U}(3)_{L} \times \mathrm{U}(3)_{E}$, and has appropriate charges under the $\mathrm{U}(1)$ factors.

Theories of FDM posit a $\mathrm{U}(3)_{\chi}$ flavor symmetry that acts on the DM field $\chi^{\alpha}$. We use lowercase Greek indices to denote the DM flavor. We focus on the case where the DM field is a fermion that transforms as a singlet under the SM gauge interactions, and has renormalizable couplings to the to the $\mathrm{SU}(2)$ singlet leptons $E_{i}^{c}$ through a scalar mediator $\phi$. The mediator does not transform under the SM and DM flavor groups. The relevant interaction takes the form

$$
\mathcal{L}_{\lambda}=\lambda_{\alpha}{ }^{i} \chi^{\alpha} E_{i}^{c} \phi+\text { h.c. }
$$

This interaction explicitly violates the $\mathrm{U}(3)_{E} \times \mathrm{U}(3)_{\chi}$ symmetry. In general, it will give rise to lepton flavor violating processes at one loop. However, when MFV is imposed, the form of this interaction is restricted, with the result that flavor violating processes are forbidden.

We impose MFV by identifying the DM flavor symmetry $\mathrm{U}(3)_{\chi}$ with the $\mathrm{U}(3)_{E}$ flavor symmetry that acts on the $\mathrm{SU}(2)$ singlet leptons in the $\mathrm{SM},{ }^{1}$ and requiring that the new interaction be invariant under the spurious $\mathrm{U}(3)^{5}$ symmetry. This leads to a restriction on the form of $\lambda_{\alpha}{ }^{j} \equiv \lambda_{i}{ }^{j}$. To leading order in $Y_{A}{ }^{i}$ we then have,

$$
\lambda_{i}^{j}=\widehat{\lambda} \delta_{i}^{j}+\tilde{\lambda} Y_{i}^{\dagger A} Y_{A}{ }^{j} .
$$

MFV also governs the form of the DM mass matrix. If $\chi$ is a Dirac fermion we can write the mass term in the Lagrangian as

$$
\mathcal{L}_{\mathrm{M}}=m_{\alpha}{ }^{\beta} \bar{\chi}_{\beta} \chi^{\alpha} \equiv m_{i}^{j} \bar{\chi}_{j} \chi^{i}
$$

MFV restricts the mass matrix to be of the form

$$
m_{i}^{j}=\widehat{m} \delta_{i}^{j}+\widetilde{m} Y_{i}^{\dagger A} Y_{A}^{j} .
$$

Then, in a basis where the lepton mass matrix is diagonal, we see that the splittings between the different DM mass eigenstates are governed by the SM Yukawa couplings in

\footnotetext{
${ }^{1}$ One could also identify $\mathrm{U}(3)_{\chi}$ with the $\mathrm{U}(3)_{L}$ symmetry that acts on the $\mathrm{SU}(2)$ doublet leptons (see [2]), but the main results do not depend on this choice.
} 
accordance with eq. (1.1). Our interest is in the scenario where the parameter $\tilde{m}$, which controls the mass splittings, is about a loop factor smaller than the parameter $m$, which sets the scale of the DM mass. Then, for DM masses of order the weak scale, the splittings can be less than an $\mathrm{MeV}$, allowing all three DM flavors to be stable on cosmological time scales.

\section{A benchmark model}

In this section we construct a simplified benchmark model which exhibits the features described above and consider its phenomenology in detail. We choose the DM candidate $\chi$ to be a Dirac fermion which transforms as a fundamental under the SM flavor group $\mathrm{SU}(3)_{E}$. The only additional terms in the Lagrangian beyond the SM are given by eq. (2.2) and (2.4). We assume that the structure of the coupling and mass terms is restricted by MFV, and follows eq. (2.3) and (2.5). For this benchmark we will further restrict to the special case where the DM mass terms and couplings generated at the high flavor scale $\Lambda$ are universal, so that $\tilde{m}$ and $\tilde{\lambda}$ are zero at this scale. ${ }^{2}$ The mass differences between the different DM flavors are then radiatively generated at one loop. The largest splittings will lie in the $\mathrm{keV}-\mathrm{MeV}$ range for WIMP DM, allowing all three flavors to contribute to the observed abundance. The mass and interaction terms for the DM in four-component notation at scale $\Lambda$ are then given by,

$$
\mathcal{L}=m_{\chi} \bar{\chi}_{i} \chi^{i}+\left[\frac{\lambda}{2} \bar{\chi}_{i}\left(1+\gamma^{5}\right) e^{i} \phi^{\dagger}+\text { h.c. }\right] .
$$

Here $e^{i}$ is the four-component spinor corresponding to the charged leptons of the SM. The only free parameters in this model are the masses of the DM and the mediator, and a coupling $\lambda$. As we shall see, the mass splittings generated in this case are finite at one loop. Later we will introduce a tiny source of flavor violation.

In what follows we determine the splittings between the different DM flavors, and show that all three flavors are stable on cosmological time scales. We then determine the range of parameters consistent with the observed relic abundance of DM. This model is constrained by a number of experiments. Constraints from $g-2$ of the muon and monophoton searches tend to be subdominant to direct detection constraints [19, 22]. LHC constraints on the production of the mediator $\phi$ and indirect detection constraints from DM annihilations into positrons and photons can also be relevant. We study these bounds in turn. Finally, we introduce a small source of flavor violation, obtain expressions for the lifetimes of the heavier flavors, and show that this naturally leads to the couplet feature.

\subsection{Mass splittings}

The breaking of the lepton flavor symmetry $\mathrm{U}(3) \rightarrow \mathrm{U}(1)^{3}$ by the SM lepton Yukawa couplings is communicated to the DM sector through the FDM interaction, eq. (2.2). Even

\footnotetext{
${ }^{2}$ Note that allowing non-vanishing $\tilde{m}$ and $\tilde{\lambda}$ at the scale $\Lambda$ would not qualitatively affect our results, provided that the parameter $\tilde{m}$ is small enough that the mass splittings between the different DM flavors are less than an MeV. In particular, the predictions for the ratios of the mass splittings, eqs. (1.4) and (1.5), do not depend on this assumption.
} 


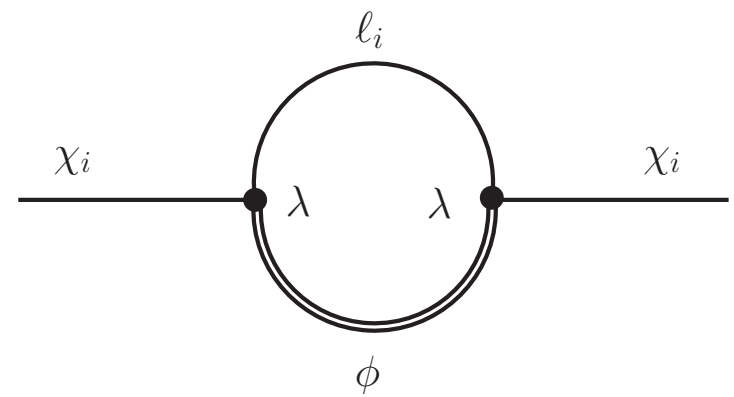

Figure 1. One-loop correction to the two-point function of $\chi$.

though they are assumed to be degenerate at tree level, mass splittings between the three $\chi$ flavors will be induced at loop level. In particular, let us consider the 2-point function for the three flavors of $\chi$ (see figure 1 ). The one-loop contributions are identical in the limit of massless leptons, but at order $\mathcal{O}\left(m_{\ell}^{2}\right)$ they begin to differ, giving rise to differences in the wavefunction renormalizations for the $\chi_{i}$. Due to the chiral nature of the FDM coupling, it is easy to see that there is no direct mass renormalization. Once the fields $\chi_{i}$ are brought back to canonical normalization, however, a mass splitting is induced between them,

$$
m_{\chi, i}-m_{\chi, j} \equiv \Delta m_{i j}=\frac{m_{\chi} \lambda^{2}}{32 \pi^{2}} \int_{0}^{1} d x x \log \left(\frac{x m_{\phi}^{2}+(1-x) m_{\ell, i}^{2}-x(1-x) m_{\chi}^{2}}{x m_{\phi}^{2}+(1-x) m_{\ell, j}^{2}-x(1-x) m_{\chi}^{2}}\right) .
$$

To leading order in the Yukawa couplings this yields,

$$
\frac{\Delta m_{i j}}{m_{\chi}} \simeq \frac{\lambda^{2}\left(y_{i}^{2}-y_{j}^{2}\right)}{64 \pi^{2}} \frac{v^{2}}{m_{\phi}^{2}} F\left(m_{\chi}^{2} / m_{\phi}^{2}\right)
$$

where as before, $y_{i}$ denote the Yukawa couplings of lepton $i$, and $v=246 \mathrm{GeV}$ is the Higgs vacuum expectation value. The loop function $F(x)$ is given by,

$$
F(x)=-\frac{x+\log (1-x)}{x^{2}} \simeq \frac{1}{2}+\frac{x}{3}+\mathcal{O}\left(x^{2}\right) .
$$

We see that $\chi_{\tau}$ is split significantly further from $\chi_{e}$ and $\chi_{\mu}$ than these two states are split from each other. For an overall mass scale $m_{\chi}$ in the $\mathrm{GeV}$ regime and $m_{\phi} \sim \mathcal{O}(100) \mathrm{GeV}$,

$$
\begin{aligned}
m_{\chi, \tau}-m_{\chi, \mu} \simeq & m_{\chi, \tau}-m_{\chi, e} \equiv \Delta m \sim \mathrm{keV} \\
& m_{\chi, \mu}-m_{\chi, e} \equiv \delta m \sim \mathrm{eV} .
\end{aligned}
$$

It is interesting to note that the one-loop splitting calculated above is a finite effect, suppressed by $v^{2} / m_{\phi}^{2}$ for large $\phi$ masses. Note that the sign of $\Delta m$ is not arbitrary, and as a consequence $\chi_{\tau}$ is the heaviest DM flavor. At two loops there arises a logarithmically divergent contribution to the mass splitting, where the log divergence is cut off at the UV flavor scale, $\Lambda$. This two-loop effect can become important for very large $m_{\phi}$ and $\Lambda$. We estimate that it is subleading to the finite one-loop calculation for the range of $m_{\phi}$ we are interested in, provided the new physics scale $\Lambda$ is less than $100 \mathrm{TeV}$. 

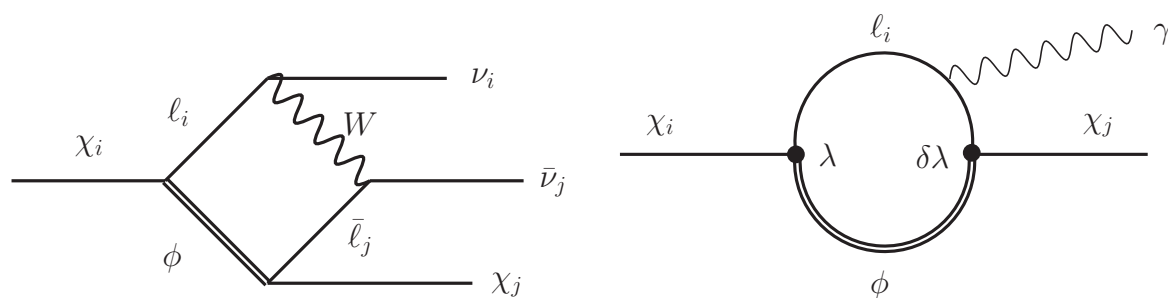

Figure 2. a) Flavor preserving decay $\chi_{i} \rightarrow \chi_{j} \nu_{i} \bar{\nu}_{j}$. b) A Feynman diagram contributing to the flavor violating decay $\chi_{i} \rightarrow \chi_{j} \gamma$.

Once the $\chi$ masses are split by these loop corrections, only the lightest $\chi$ is truly stable. This is true even in the exact MFV limit where the $\mathrm{U}(1)^{3}$ flavor symmetry is preserved. For this benchmark, the splittings are smaller than the mass of the electron. Then, the leading contribution for flavor-preserving $\chi$ decays arises at one loop and is illustrated in the left panel of figure 2. Note that this contribution is very suppressed due to the following three factors.

- With a $\chi$ mass splitting of order $\mathrm{keV}$, the kinematically available phase space is extremely small. This results in a significant suppression for the $1 \rightarrow 3$ process.

- The loop amplitude is suppressed by the momentum-exchange scale, or more concretely by $\left(\Delta m / m_{\phi}\right)$.

- The lepton propagators in the loop couple to $\phi$ on one end and to $W^{ \pm}$on the other. However, the former couples to right-chirality leptons while the latter couples to leftchirality leptons. Therefore both lepton propagators need a mass insertion to obtain a nonzero amplitude, so the decay rate is further suppressed by $m_{\ell_{i}}^{2} m_{\ell_{j}}^{2}$.

As a consequence of these effects, heavier flavors are long-lived on cosmological time scales.

\subsection{Relic abundance}

The DM annihilation rate in each channel $\left(\chi_{i} \bar{\chi}_{j} \rightarrow \ell_{i} \bar{\ell}_{j}\right)$ is given by,

$$
\langle\sigma v\rangle=\frac{\lambda^{4} m_{\chi}^{2}}{32 \pi\left(m_{\chi}^{2}+m_{\phi}^{2}\right)^{2}} .
$$

Since the DM candidate is a Dirac fermion, there is no p-wave or chirality suppression, and thus the annihilation cross section today is the same as in the early universe to a good approximation.

Since all flavor combinations of DM co-annihilate with one another with the same cross section, the cross section that gives rise to the correct relic abundance today is the same as for a single species of Dirac fermion DM, given by

$$
\langle\sigma v\rangle=2 \times\left(2.2 \times 10^{-26} \mathrm{~cm}^{3} / \mathrm{s}\right) .
$$

The factor of two relative to the canonical quoted value (for Majorana DM) arises due to the Dirac nature of the DM particle. The region of parameter space leading to the correct relic density is shown in figure 3 as a red band. 

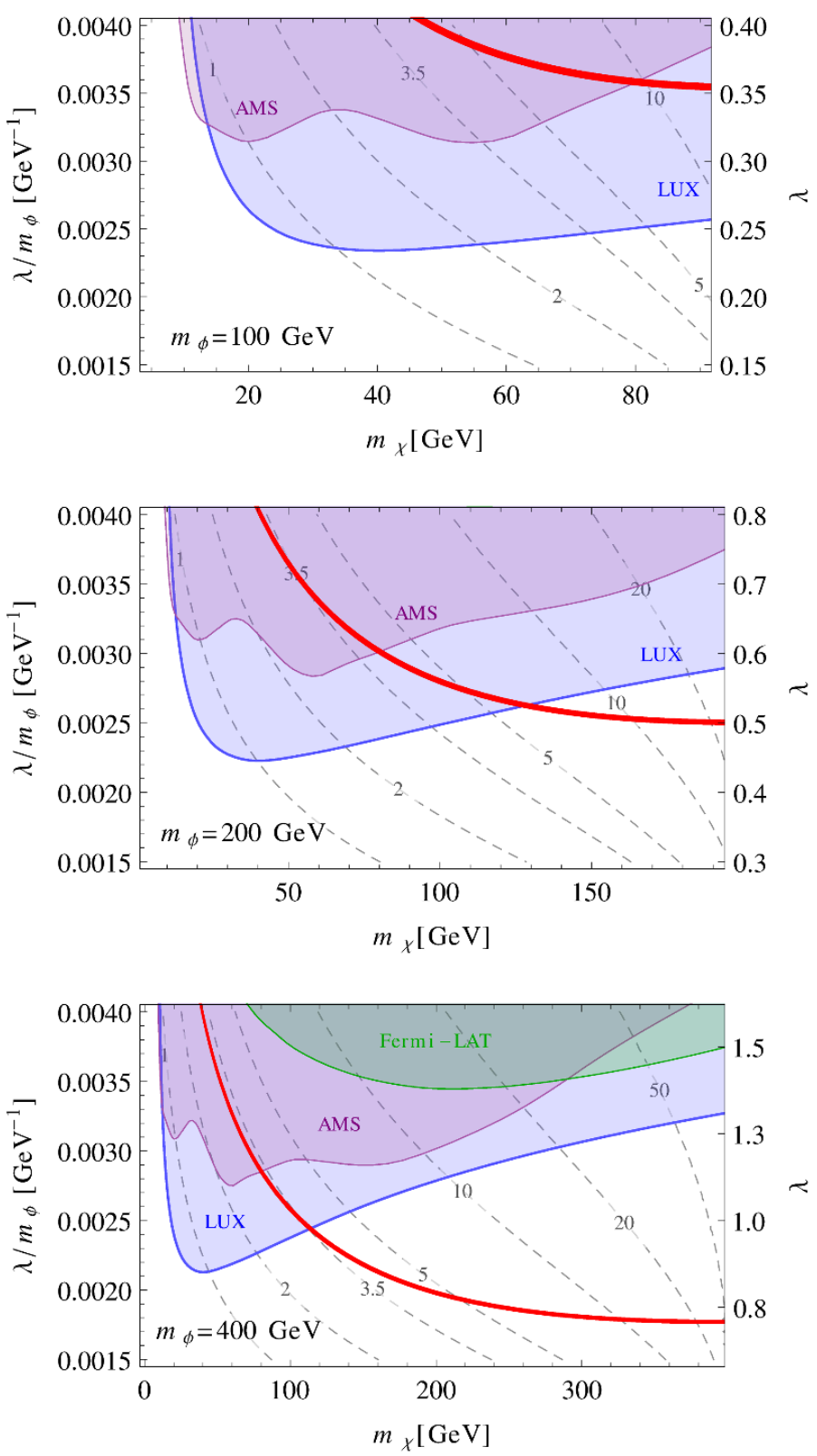

Figure 3. For mediator masses $m_{\phi}=100,200$ and $400 \mathrm{GeV}$, we plot the position of the X-ray signal (in keV, gray dashed contours) as well as a number of constraints. Direct detection constraints from LUX are shown as the blue-shaded region, while the indirect detection constraints from positrons and photons are shown as the purple and green-shaded regions, respectively. The red band shows the region where correct relic abundance is obtained. 
Note that the above calculation applies only if we assume that the interaction with leptons alone is responsible for the DM thermal relic abundance. Any coupling to additional non-SM states will alter these numbers. This constraint can also be relaxed if the DM relic density is set by an asymmetry, which can arise rather naturally in these models [9].

\subsection{Direct detection}

As discussed in detail in [2], lepton flavored DM can scatter off nuclei via a one-loop photon exchange. These constraints can be severe in the region where the DM is a thermal relic. The dominant contribution to the WIMP-nucleon cross section is flavor diagonal and for each flavor of FDM it is given by,

$$
\sigma_{n}=\frac{\mu_{n}^{2} Z^{2}}{A^{2} \pi} \sum_{\ell}\left(\frac{\lambda^{2} e^{2}}{64 \pi^{2} m_{\phi}^{2}}\left[1+\frac{2}{3} \log \frac{\Lambda_{\ell}^{2}}{m_{\phi}^{2}}\right]\right)^{2} .
$$

Here $\mu_{n}$ is the reduced mass of the DM-nucleon system and $\Lambda_{\ell}$ represents the infrared cutoff in the loop calculation for the effective DM-photon coupling. This cutoff is $m_{\ell}$, the mass of the corresponding lepton, unless $m_{\ell}$ is smaller than the momentum exchange in the process, of the order of $10 \mathrm{MeV}$. We therefore use $\Lambda_{\tau}=m_{\tau}$ and $\Lambda_{\mu}=m_{\mu}$, but set $\Lambda_{e}=10 \mathrm{MeV}$. In extracting constraints from the null results of direct detection experiments such as LUX, we use the total rate, summed over all three FDM flavors. The region of parameter space excluded by LUX is shown in figure 3 as the blue-shaded region.

\subsection{Indirect detection}

In the limit $m_{\chi} \ll m_{\phi},(\Delta m)^{2}$ and $\langle\sigma v\rangle$ both scale approximately as $m_{\chi}^{2} \lambda^{4} / m_{\phi}^{4}$, and therefore choosing a fixed mass splitting or requiring thermal relic abundance leads to potentially observable signals for indirect detection searches in photons and positrons (with the caveats mentioned at the end of the previous paragraph). The constraints from both indirect detection channels are more stringent for lower mass DM, since the signal rate scales as the square of the $\chi$ number density, which itself scales as $m_{\chi}^{-1}$, while the background flux as a function of energy does not change as rapidly. Therefore, for a given $\Delta m$ or $\langle\sigma v\rangle$, these constraints can be weakened by increasing the DM mass and either decreasing the coupling or increasing $m_{\phi}$.

For the positron constraint from the AMS-02 experiment [23], the signal has contributions both from the prompt positrons produced when one of the annihilating particles is $\bar{\chi}_{e}$, and also from secondary positrons from the decays of $\mu^{+}$and $\tau^{+}$that are produced when one of the annihilating particles is $\bar{\chi}_{\mu}$ or $\bar{\chi}_{\tau}$. The spectrum of the secondary positrons is shifted towards lower energies compared to the prompt positrons (not to mention that the branching ratio of $\tau \rightarrow e+X$ is rather low), and therefore the bound from AMS-02 comes mostly from the prompt positrons. The bound is shown in figure 3 as the purple-shaded region. Note that the positron constraint is significantly weaker than the constraint from direct detection across the parameter space, and therefore the inclusion or non-inclusion of secondary positrons in determining the bound turns out to be academic. Owing to the relative factor of two between Dirac and Majorana DM (eq. (3.8)), the latter being relevant 
for SUSY for which the AMS bounds are calculated, the bound on the FDM annihilation cross section leading from prompt positrons (any one of the three $\chi_{\ell}$ flavors annihilating with $\bar{\chi}_{e}$ ) is related to the total annihilation cross section (all nine annihilation channels) as

$$
\langle\sigma v\rangle \leq 6\langle\sigma v\rangle_{\text {bound }, e^{+}}
$$

where $\langle\sigma v\rangle_{\text {bound, } e^{+}}$is the experimental bound quoted in [23] for a Majorana DM annihilating to $e^{+} e^{-}$with $100 \%$ branching fraction.

Similar to the case of positron constraints being most sensitive to prompt positrons in the final state, indirect detection in photons is most sensitive to $\tau$ 's in the final state, since more photons are produced from $\tau$ 's than from $e$ 's or $\mu$ 's. One can therefore formulate the bound from indirect detection in the photon final state [24] in terms of the effective annihilation cross section leading to the production of $\tau$ 's,

$$
\langle\sigma v\rangle \leq 6\langle\sigma v\rangle_{\text {bound }, \gamma}
$$

where $\langle\sigma v\rangle_{\text {bound, } \gamma}$ is the experimental bound quote in [24] for a Majorana DM annihilating to $\tau^{+} \tau^{-}$with $100 \%$ branching ratio. The constraint from indirect detection in photons is shown in figure 3 as the green-shaded region.

DM annihilating to leptons can potentially have significant constraints from the CMB $[25,26]$. However, the annihilation into muons and taus has a low efficiency to inject energy into the CMB [27], and the constraints are subdominant to the other constraints considered above.

\subsection{The couplet}

Since the rates of flavor-preserving $\chi$ decays are so extremely small, even a very small flavor-violating contribution can easily be the dominant channel for the decays of the heavier $\chi$. Such decays could, for example, arise from the flavor violating dipole operator, $\bar{\chi}_{i} \sigma_{\mu \nu} \chi_{j} F^{\mu \nu}$, which generates the process $\chi_{i} \rightarrow \chi_{j}+\gamma$. The monochromatic X-ray photons from these transitions would then constitute a striking signal of this scenario, and exhibit the couplet feature. The dipole operator is, however, non-renormalizable. We can obtain the same effect at the renormalizable level by adding to the Lagrangian a tiny flavor violating contribution to the DM-visible matter interaction term,

$$
\mathcal{L}_{\mathrm{FV}}=\frac{1}{2} \delta \lambda_{i j} \bar{\chi}_{i}\left(1+\gamma^{5}\right) e_{j} \phi^{\dagger}+\text { h.c. }
$$

This radiatively generates the flavor violating dipole transition $\chi_{i} \rightarrow \chi_{j}+\gamma$ as illustrated in the right panel of figure 2. Note that unlike the flavor-preserving decays, these are two-body decays, and there are no suppressions due to lepton masses. The rate for these flavor-violating decays can be calculated in a straightforward manner. If we assume that all off-diagonal couplings in $\delta \lambda_{i j}$ are of the same size $\delta \lambda \ll \lambda$, then to leading order

$$
\Gamma_{i j} \equiv \Gamma_{\chi_{i} \rightarrow \chi_{j} \gamma}=\frac{e^{2} \lambda^{2} \delta \lambda^{2}}{1024 \pi^{5}} \frac{\left(\Delta m_{i j}\right)^{3} m_{\chi}^{2}}{m_{\phi}^{4}},
$$


where we have neglected higher order terms proportional to lepton masses. We see that $\Gamma_{i j} \propto\left(\Delta m_{i j}\right)^{3}$. Then, in the absence of any hierarchy in the $\delta \lambda$, it follows that the rates for the $\chi_{\tau} \rightarrow \chi_{\mu}$ and $\chi_{\tau} \rightarrow \chi_{e}$ transitions are comparable. However, transitions between $\chi_{\mu}$ and $\chi_{e}$ will be many orders of magnitude slower than this, and are not expected to be be observable.

The parameters $\delta \lambda$ allow the heaviest flavor to decay on cosmological timescales. The current sensitivity for dark matter decay lifetimes from X-ray observations in the keV-MeV range is roughly at the level of $\tau \sim 10^{27}-10^{28} \sec [28,29]$. Having restricted the parameters $\lambda, m_{\chi}$ and $m_{\phi}$ to satisfy the relic abundance condition eq. (3.7), this can be translated into a bound on the flavor vioating coupling $\delta \lambda$, expressed in terms of the splitting $\Delta m$ between $\chi_{\tau}$ and the two lighter flavors,

$$
\frac{\delta \lambda}{\lambda} \lesssim 10^{-7}\left(\frac{\mathrm{keV}}{\Delta m}\right)^{2} \sqrt{\frac{m_{\chi}}{100 \mathrm{GeV}}} .
$$

It follows that, in the allowed range, $\delta \lambda$ is much too small to affect the relic abundance calculation, or any of the bounds on this scenario considered in the previous sections. It remains to verify that the contribution of the $\delta \lambda$ to the DM mass splittings is subdominant to the previously calculated splittings associated with the SM Yukawa couplings, eq. (3.3). The off-diagonal entries in the coupling matrix responsible for the flavor-violating decay only affect the mass eigenvalues at quadratic order in $\delta \lambda$. Consequently, once the bound eq. (3.14) is imposed, this effect is much smaller than the splitting associated with the SM Yukawa couplings calculated in eq. (3.3). However, in the absence of a symmetry that restricts the form of $\delta \lambda$, we expect that the diagonal elements of this matrix will be parametrically of the same size as the off-diagonal flavor-violating entries. These diagonal terms will, in general, not be flavor universal, and will contribute to the mass eigenvalues at linear order in $\delta \lambda$. Therefore, it is important to understand whether this effect can dominate over the splitting calculated in eq. (3.3). At one loop there is a logarithmically enhanced contribution from $\delta \lambda$ that is cutoff at the UV flavor scale $\Lambda$,

$$
\frac{\delta m_{\chi}}{m_{\chi}}=\frac{\lambda \delta \lambda}{8 \pi^{2}} \int_{0}^{1} d x x \log \left[\frac{\left(x m_{\phi}^{2}-x(1-x) m_{\chi}^{2}\right)}{\Lambda^{2}}\right] .
$$

For $\delta \lambda$ satisfying the bound, eq. (3.14), this effect is always much smaller than the splitting between $\chi_{\tau}$ and the other flavors, showing that the existence of the couplet is a robust prediction of this framework. However, for $\delta \lambda / \lambda \gtrsim 10^{-8}$, corresponding to $\Delta m \lesssim 10 \mathrm{keV}$, this splitting can become comparable to or larger than the splitting between $\chi_{e}$ and $\chi_{\mu}$ from eq. (3.3). Hence the prediction for the splitting between the lines in the couplet, eq. (1.5), is only valid if the mass difference between $\chi_{\tau}$ and the other flavors is larger than about $10 \mathrm{keV}$.

\subsection{The $3.5 \mathrm{keV}$ line}

An X-ray line signal has been observed at $3.5 \mathrm{keV}[30,31]$. We note that there is currently no consensus about the interpretation of this observation as arising from a DM signal [3243]. Nevertheless, a large number of DM models have been proposed to explain this signal. 


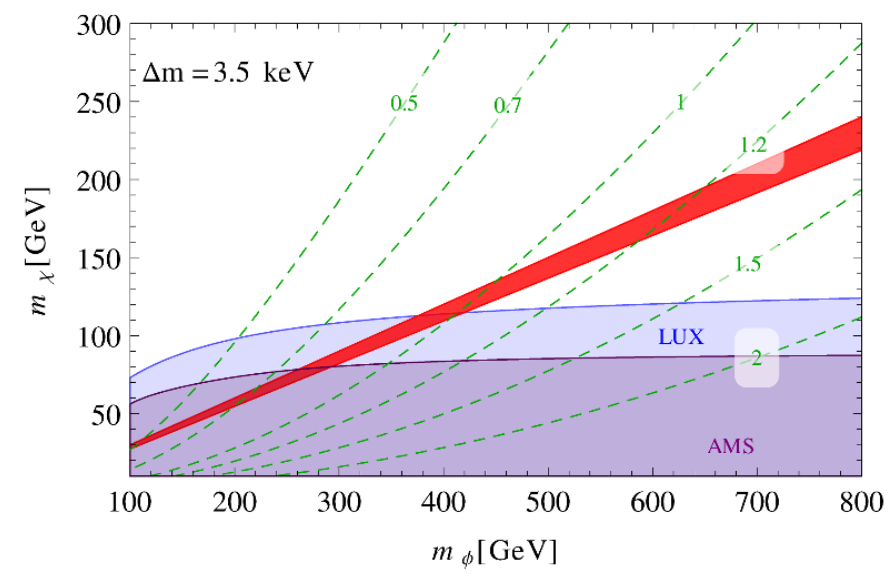

Figure 4. Constraints on the mass of DM, $m_{\chi}$ and the mediator, $m_{\phi}$ when the X-ray line is at $3.5 \mathrm{keV}$. The contours show the value of coupling $\lambda$, and the red band shows the region where correct relic abundance is obtained. The blue and purple-shaded regions show the exclusion from LUX and from AMS respectively.

The ideas that have been proposed include sterile neutrinos [44-69], axions [70-85], supersymmetry [86-92] and a number of other mechanisms [93-124]. We now show that the $3.5 \mathrm{keV}$ line, if confirmed, can be easily accommodated within the framework of the minimal model. We show in figure 3 the region of parameter space that provides the right relic abundance for DM, consistent with a $3.5 \mathrm{keV}$ line. Fixing the splitting to be $3.5 \mathrm{keV}$, we show the direct detection and the AMS positron constraint in figure 4 along with the region of parameter space consistent with the requirement of correct relic abundance.

Our scenario further predicts that closer inspection of this line will reveal two closely spaced lines corresponding to the $\chi_{\tau} \rightarrow \chi_{\mu}$ and $\chi_{\tau} \rightarrow \chi_{e}$ transitions. This couplet constitutes a characteristic indirect detection signal of lepton FDM scenarios. However, because the frequencies of these lines is less than $10 \mathrm{keV}$, in general the prediction for their splitting, eq. (1.5), is not expected to apply. To understand this in greater detail, note that the lifetime for a decaying DM candidate to be consistent with the observed signal is given by (see for instance [99]),

$$
\tau_{\mathrm{DM}} \simeq\left(10^{28} \mathrm{sec}\right) \frac{7 \mathrm{keV}}{m_{\chi}}
$$

Then, for $m_{\chi}=150 \mathrm{GeV}$, one obtains $\tau_{\mathrm{DM}} \approx 10^{20} \mathrm{~s}$. For $m_{\phi}=500 \mathrm{GeV}$ and $\lambda \simeq 1$, this would require $\delta \lambda \simeq 10^{-8}$. Then the contribution to the splittings from the flavor diagonal elements of the matrix $\delta \lambda$, given by eq. (3.15), can comparable to or larger than the small mass splitting between the two light DM flavors that arises from the SM Yukawa couplings, eq. (3.3). Therefore, eq. (1.5) is not expected to apply. However, the contribution from $\delta \lambda$ is still much smaller than the large $3.5 \mathrm{keV}$ splitting between $\chi_{\tau}$ and the other two flavors. Consequently, the characteristic couplet feature survives. 


\section{Conclusions}

We have studied models of lepton FDM within the MFV framework. In this scenario, mass splittings between different DM flavors can naturally be small enough that tree level decays of heavy flavors are kinematically forbidden. Then all three flavors of DM can be long-lived on cosmological time scales (the lightest flavor being exactly stable). The ratios of the mass splittings between the three possible pairings among the DM flavors are predicted.

When even a very small source of flavor violation is present in the dark sector, a new decay channel becomes available for the decay of heavier DM flavors through a dipole transition. While the lifetime associated with this decay may be orders of magnitude longer than the age of the universe, it can still be the dominant decay channel and gives rise to a very distinct final state. These decays result in two very closely separated photon lines, the couplet. For weak scale DM, the overall energy of the couplet is naturally in the keV-MeV range, with the splitting of the two lines in the eV-keV range.

We have focused on the detailed phenomenology of a specific model of lepton flavored DM. In this model, the mass splittings are radiatively generated by finite one loop effects arising from the breaking of the lepton flavor symmetry by Yukawa couplings. The sign of the contribution is fixed, with the result that $\chi_{\tau}$ is the heaviest and $\chi_{e}$ the lightest state.

This scenario is a potential explanation for the claimed observation of a $3.5 \mathrm{keV}$ line in the X-ray spectrum, and there exist regions in parameter space of the model where such an explanation is entirely consistent with the observed DM relic density as well as with experimental constraints set by a number of direct and indirect detection experiments. While a DM explanation of this line is in dispute, the characteristic double-line structure predicted by the couplet can be directly tested experimentally. In particular, the next generation experiments might be able to resolve any such feature in the X-ray spectrum.

\section{Acknowledgments}

We thank Ilias Cholis and Dan Hooper for useful discussions. CK would also like to thank the Aspen Center for Physics (supported by the National Science Foundation under Grant No. PHYS-1066293) as well as the Perimeter Institute for Theoretical Physics (supported by the Government of Canada through Industry Canada and by the Province of Ontario through the Ministry of Research and Innovation), where part of this work was completed, for their hospitality. ZC and CV are supported by NSF under grant PHY-1315155. CK is supported by NSF grant numbers PHY-1315983 and PHY-1316033. Fermilab is operated by Fermi Research Alliance, LLC under Contract No. De-AC02-07CH11359 with the United States Department of Energy.

Open Access. This article is distributed under the terms of the Creative Commons Attribution License (CC-BY 4.0), which permits any use, distribution and reproduction in any medium, provided the original author(s) and source are credited. 


\section{References}

[1] B. Batell, J. Pradler and M. Spannowsky, Dark Matter from Minimal Flavor Violation, JHEP 08 (2011) 038 [arXiv:1105.1781] [INSPIRE].

[2] P. Agrawal, S. Blanchet, Z. Chacko and C. Kilic, Flavored Dark Matter and Its Implications for Direct Detection and Colliders, Phys. Rev. D 86 (2012) 055002 [arXiv:1109.3516] [INSPIRE].

[3] A. Kumar and S. Tulin, Top-flavored dark matter and the forward-backward asymmetry, Phys. Rev. D 87 (2013) 095006 [arXiv:1303.0332] [INSPIRE].

[4] L. Lopez-Honorez and L. Merlo, Dark matter within the minimal flavour violation ansatz, Phys. Lett. B 722 (2013) 135 [arXiv:1303.1087] [INSPIRE].

[5] J. Kile, Flavored Dark Matter: A Review, Mod. Phys. Lett. A 28 (2013) 1330031 [arXiv: 1308.0584$]$ [INSPIRE].

[6] B. Batell, T. Lin and L.-T. Wang, Flavored Dark Matter and R-Parity Violation, JHEP 01 (2014) 075 [arXiv: 1309.4462] [INSPIRE].

[7] P. Agrawal, B. Batell, D. Hooper and T. Lin, Flavored Dark Matter and the Galactic Center Gamma-Ray Excess, Phys. Rev. D 90 (2014) 063512 [arXiv:1404.1373] [InSPIRE].

[8] P. Agrawal, M. Blanke and K. Gemmler, Flavored dark matter beyond Minimal Flavor Violation, JHEP 10 (2014) 72 [arXiv: 1405.6709] [INSPIRE].

[9] A. Hamze, C. Kilic, J. Koeller, C. Trendafilova and J.-H. Yu, Lepton-Flavored Asymmetric Dark Matter and Interference in Direct Detection, Phys. Rev. D 91 (2015) 035009 [arXiv: 1410.3030] [INSPIRE].

[10] C.-J. Lee and J. Tandean, Lepton-Flavored Scalar Dark Matter with Minimal Flavor Violation, JHEP 04 (2015) 174 [arXiv: 1410.6803] [inSPIRE].

[11] C. Kilic, M.D. Klimek and J.-H. Yu, Signatures of Top Flavored Dark Matter, Phys. Rev. D 91 (2015) 054036 [arXiv: 1501.02202] [INSPIRE].

[12] J. Kile and A. Soni, Flavored Dark Matter in Direct Detection Experiments and at LHC, Phys. Rev. D 84 (2011) 035016 [arXiv:1104.5239] [INSPIRE].

[13] J.F. Kamenik and J. Zupan, Discovering Dark Matter Through Flavor Violation at the LHC, Phys. Rev. D 84 (2011) 111502 [arXiv:1107.0623] [INSPIRE].

[14] Y. Zhang, Top Quark Mediated Dark Matter, Phys. Lett. B 720 (2013) 137 [arXiv:1212.2730] [INSPIRE].

[15] J. Kile, A. Kobach and A. Soni, Lepton-Flavored Dark Matter, Phys. Lett. B 744 (2015) 330 [arXiv:1411.1407] [INSPIRE].

[16] J. March-Russell, C. McCabe and M. McCullough, Neutrino-Flavoured Sneutrino Dark Matter, JHEP 03 (2010) 108 [arXiv: 0911.4489] [INSPIRE].

[17] Y. Bai and J. Berger, Lepton Portal Dark Matter, JHEP 08 (2014) 153 [arXiv:1402.6696] [INSPIRE].

[18] S. Chang, R. Edezhath, J. Hutchinson and M. Luty, Leptophilic Effective WIMPs, Phys. Rev. D 90 (2014) 015011 [arXiv: 1402.7358] [InSPIRE].

[19] P. Agrawal, Z. Chacko and C.B. Verhaaren, Leptophilic Dark Matter and the Anomalous Magnetic Moment of the Muon, JHEP 08 (2014) 147 [arXiv:1402.7369] [INSPIRE]. 
[20] T. Takahashi et al., The ASTRO-H Mission, Proc. SPIE Int. Soc. Opt. Eng. 7732 (2010) $77320 Z$ [arXiv: 1010.4972] [INSPIRE].

[21] T. Takahashi et al., ASTRO-H White Paper - Introduction, arXiv:1412.2351 [INSPIRE].

[22] A. Freitas, J. Lykken, S. Kell and S. Westhoff, Testing the Muon g-2 Anomaly at the LHC, JHEP 05 (2014) 145 [Erratum ibid. 09 (2014) 155] [arXiv: 1402.7065] [INSPIRE].

[23] A. Ibarra, A.S. Lamperstorfer and J. Silk, Dark matter annihilations and decays after the AMS-02 positron measurements, Phys. Rev. D 89 (2014) 063539 [arXiv:1309.2570] [INSPIRE].

[24] M. Tavakoli, I. Cholis, C. Evoli and P. Ullio, Constraints on dark matter annihilations from diffuse gamma-ray emission in the Galaxy, JCAP 01 (2014) 017 [arXiv:1308.4135] [INSPIRE].

[25] N. Padmanabhan and D.P. Finkbeiner, Detecting dark matter annihilation with CMB polarization: Signatures and experimental prospects, Phys. Rev. D 72 (2005) 023508 [astro-ph/0503486] [INSPIRE].

[26] T.R. Slatyer, N. Padmanabhan and D.P. Finkbeiner, CMB Constraints on WIMP Annihilation: Energy Absorption During the Recombination Epoch, Phys. Rev. D 80 (2009) 043526 [arXiv:0906.1197] [INSPIRE].

[27] M.S. Madhavacheril, N. Sehgal and T.R. Slatyer, Current Dark Matter Annihilation Constraints from CMB and Low-Redshift Data, Phys. Rev. D 89 (2014) 103508 [arXiv:1310.3815] [INSPIRE].

[28] A. Boyarsky, D. Iakubovskyi, O. Ruchayskiy and V. Savchenko, Constraints on decaying Dark Matter from XMM-Newton observations of M31, Mon. Not. Roy. Astron. Soc. 387 (2008) 1361 [arXiv: 0709.2301] [INSPIRE].

[29] J.W. den Herder et al., The Search for decaying Dark Matter, arXiv:0906.1788 [INSPIRE].

[30] E. Bulbul, M. Markevitch, A. Foster, R.K. Smith, M. Loewenstein and S.W. Randall, Detection of An Unidentified Emission Line in the Stacked X-ray spectrum of Galaxy Clusters, Astrophys. J. 789 (2014) 13 [arXiv:1402.2301] [INSPIRE].

[31] A. Boyarsky, O. Ruchayskiy, D. Iakubovskyi and J. Franse, Unidentified Line in X-Ray Spectra of the Andromeda Galaxy and Perseus Galaxy Cluster, Phys. Rev. Lett. 113 (2014) 251301 [arXiv: 1402.4119] [INSPIRE].

[32] S. Riemer-Sorensen, Questioning a $3.5 \mathrm{keV}$ dark matter emission line, arXiv:1405.7943 [INSPIRE].

[33] A. Boyarsky, J. Franse, D. Iakubovskyi and O. Ruchayskiy, Checking the dark matter origin of $3.53 \mathrm{keV}$ line with the Milky Way center, arXiv:1408.2503 [INSPIRE].

[34] T.E. Jeltema and S. Profumo, Discovery of a $3.5 \mathrm{keV}$ line in the Galactic Centre and a critical look at the origin of the line across astronomical targets, Mon. Not. Roy. Astron. Soc. 450 (2015) 2143 [arXiv:1408.1699] [INSPIRE].

[35] D. Malyshev, A. Neronov and D. Eckert, Constraints on $3.55 \mathrm{keV}$ line emission from stacked observations of dwarf spheroidal galaxies, Phys. Rev. D 90 (2014) 103506 [arXiv: 1408.3531] [INSPIRE].

[36] M.E. Anderson, E. Churazov and J.N. Bregman, Non-Detection of X-Ray Emission From Sterile Neutrinos in Stacked Galaxy Spectra, arXiv:1408.4115 [INSPIRE]. 
[37] A. Boyarsky, J. Franse, D. Iakubovskyi and O. Ruchayskiy, Comment on the paper "Dark matter searches going bananas: the contribution of Potassium (and Chlorine) to the $3.5 \mathrm{keV}$ line" by T. Jeltema and S. Profumo, arXiv:1408.4388 [inSPIRE].

[38] E. Bulbul, M. Markevitch, A.R. Foster, R.K. Smith, M. Loewenstein and S.W. Randall, Comment on "Dark matter searches going bananas: the contribution of Potassium (and Chlorine) to the $3.5 \mathrm{keV}$ line", arXiv:1409.4143 [INSPIRE].

[39] T. Jeltema and S. Profumo, Reply to Two Comments on "Dark matter searches going bananas the contribution of Potassium (and Chlorine) to the $3.5 \mathrm{keV}$ line", arXiv: 1411.1759 [INSPIRE].

[40] D. Iakubovskyi, New emission line at $\sim 3.5 \mathrm{keV}$ - observational status, connection with radiatively decaying dark matter and directions for future studies, arXiv:1410.2852 [INSPIRE].

[41] E. Carlson, T. Jeltema and S. Profumo, Where do the $3.5 \mathrm{keV}$ photons come from? A morphological study of the Galactic Center and of Perseus, JCAP 02 (2015) 009 [arXiv: 1411.1758] [INSPIRE].

[42] T. Tamura, R. Iizuka, Y. Maeda, K. Mitsuda and N.Y. Yamasaki, An X-ray Spectroscopic Search for Dark Matter in the Perseus Cluster with Suzaku, Publ. Astron. Soc. Jap. 67 (2015) 23 [arXiv:1412.1869] [INSPIRE].

[43] O. Urban, N. Werner, S.W. Allen, A. Simionescu, J.S. Kaastra and L.E. Strigari, A Suzaku Search for Dark Matter Emission Lines in the X-ray Brightest Galaxy Clusters, Mon. Not. Roy. Astron. Soc. 451 (2015) 2447 [arXiv: 1411.0050] [INSPIRE].

[44] S. Patra, N. Sahoo and N. Sahu, Dipolar dark matter in light of the $3.5 \mathrm{keV}$ x-ray line, neutrino mass and LUX data, Phys. Rev. D 91 (2015) 115013 [arXiv:1412.4253] [INSPIRE].

[45] M. Lattanzi, R.A. Lineros and M. Taoso, Connecting neutrino physics with dark matter, New J. Phys. 16 (2014) 125012 [arXiv:1406.0004] [InSPIRE].

[46] N. Haba, H. Ishida and R. Takahashi, $\nu_{R}$ dark matter-philic Higgs for $3.5 \mathrm{keV} X$-ray signal, Phys. Lett. B 743 (2015) 35 [arXiv:1407.6827] [INSPIRE].

[47] B. Shuve and I. Yavin, Dark matter progenitor: Light vector boson decay into sterile neutrinos, Phys. Rev. D 89 (2014) 113004 [arXiv:1403.2727] [INSPIRE].

[48] W. Rodejohann and H. Zhang, Signatures of Extra Dimensional Sterile Neutrinos, Phys. Lett. B 737 (2014) 81 [arXiv:1407.2739] [INSPIRE].

[49] A. Abada, V. De Romeri and A.M. Teixeira, Effect of steriles states on lepton magnetic moments and neutrinoless double beta decay, JHEP 09 (2014) 074 [arXiv:1406.6978] [INSPIRE].

[50] S. Baek and H. Okada, 7 keV Dark Matter as X-ray Line Signal in Radiative Neutrino Model, arXiv:1403.1710 [INSPIRE].

[51] S. Chakraborty, A. Datta and S. Roy, $h \rightarrow \gamma \gamma$ in $\mathrm{U}(1)_{R}$-lepton number model with a right-handed neutrino, JHEP 02 (2015) 124 [arXiv:1411.1525] [INSPIRE].

[52] Z. Kang, FImP Miracle of Sterile Neutrino Dark Matter by Scale Invariance, arXiv: 1411.2773 [INSPIRE]. 
[53] A.A. Dobrynina, N.V. Mikheev and G.G. Raffelt, Radiative decay of keV-mass sterile neutrinos in a strongly magnetized plasma, Phys. Rev. D 90 (2014) 113015 [arXiv:1410.7915] [INSPIRE].

[54] H. Okada and T. Toma, 3.55 keV X-ray Line Signal from Excited Dark Matter in Radiative Neutrino Model, Phys. Lett. B 737 (2014) 162 [arXiv:1404.4795] [INSPIRE].

[55] H. Okada and Y. Orikasa, X-ray line in Radiative Neutrino Model with Global U(1) Symmetry, Phys. Rev. D 90 (2014) 075023 [arXiv: 1407.2543] [INSPIRE].

[56] A. Abada, G. Arcadi and M. Lucente, Dark Matter in the minimal Inverse Seesaw mechanism, arXiv:1406.6556 [INSPIRE].

[57] H. Ishida and H. Okada, $3.55 \mathrm{keV}$ X-ray Line Interpretation in Radiative Neutrino Model, arXiv: 1406.5808 [INSPIRE].

[58] J.M. Cline, Y. Farzan, Z. Liu, G.D. Moore and W. Xue, $3.5 \mathrm{keV} x$ rays as the "21 cm line" of dark atoms and a link to light sterile neutrinos, Phys. Rev. D 89 (2014) 121302 [arXiv: 1404.3729] [INSPIRE].

[59] K.P. Modak, 3.5 keV X-ray Line Signal from Decay of Right-Handed Neutrino due to Transition Magnetic Moment, JHEP 03 (2015) 064 [arXiv:1404.3676] [INSPIRE].

[60] D.J. Robinson and Y. Tsai, Dynamical framework for KeV Dirac neutrino warm dark matter, Phys. Rev. D 90 (2014) 045030 [arXiv: 1404.7118] [INSPIRE].

[61] S. Chakraborty, D.K. Ghosh and S. Roy, 7 keV Sterile neutrino dark matter in $U(1)_{R}$-lepton number model, JHEP 10 (2014) 146 [arXiv:1405.6967] [INSPIRE].

[62] A. Adulpravitchai and M.A. Schmidt, A Fresh Look at keV Sterile Neutrino Dark Matter from Frozen-In Scalars, JHEP 01 (2015) 006 [arXiv:1409.4330] [INSPIRE].

[63] S. Patra and P. Pritimita, 7 keV sterile neutrino Dark Matter in extended seesaw framework, arXiv:1409.3656 [INSPIRE].

[64] A. Merle and A. Schneider, Production of Sterile Neutrino Dark Matter and the $3.5 \mathrm{keV}$ line, arXiv:1409.6311 [INSPIRE].

[65] M. Frigerio and C.E. Yaguna, Sterile Neutrino Dark Matter and Low Scale Leptogenesis from a Charged Scalar, Eur. Phys. J. C 75 (2015) 31 [arXiv:1409.0659] [InSPIRE].

[66] T. Tsuyuki, Neutrino masses, leptogenesis and sterile neutrino dark matter, Phys. Rev. D 90 (2014) 013007 [arXiv: 1403.5053] [InSPIRE].

[67] K.N. Abazajian, Resonantly Produced 7 keV Sterile Neutrino Dark Matter Models and the Properties of Milky Way Satellites, Phys. Rev. Lett. 112 (2014) 161303 [arXiv:1403.0954] [INSPIRE].

[68] S.K. Kang and A. Patra, keV Sterile Neutrino Dark Matter and Low Scale Leptogenesis, arXiv: 1412.4899 [INSPIRE].

[69] A. Harada, A. Kamada and N. Yoshida, Structure formation in a mixed dark matter model with decaying sterile neutrino: the $3.5 \mathrm{keV} X$-ray line and the Galactic substructure, arXiv: 1412.1592 [INSPIRE].

[70] K.-Y. Choi and O. Seto, X-ray line signal from decaying axino warm dark matter, Phys. Lett. B 735 (2014) 92 [arXiv:1403.1782] [InSPIRE].

[71] S.P. Liew, Axino dark matter in light of an anomalous X-ray line, JCAP 05 (2014) 044 [arXiv: 1403.6621] [INSPIRE]. 
[72] T. Higaki, N. Kitajima and F. Takahashi, Hidden axion dark matter decaying through mixing with QCD axion and the $3.5 \mathrm{keV} X$-ray line, JCAP 12 (2014) 004 [arXiv:1408.3936] [INSPIRE].

[73] M. Kawasaki, N. Kitajima and F. Takahashi, Relaxing Isocurvature Bounds on String Axion Dark Matter, Phys. Lett. B 737 (2014) 178 [arXiv:1406.0660] [InSPIRE].

[74] T. Higaki, K.S. Jeong and F. Takahashi, The 7 keV axion dark matter and the X-ray line signal, Phys. Lett. B 733 (2014) 25 [arXiv:1402.6965] [INSPIRE].

[75] J. Jaeckel, J. Redondo and A. Ringwald, $3.55 \mathrm{keV}$ hint for decaying axionlike particle dark matter, Phys. Rev. D 89 (2014) 103511 [arXiv:1402.7335] [InSPIRE].

[76] H.M. Lee, S.C. Park and W.-I. Park, Cluster X-ray line at $3.5 \mathrm{keV}$ from axion-like dark matter, Eur. Phys. J. C 74 (2014) 3062 [arXiv:1403.0865] [INSPIRE].

[77] J.-C. Park, S.C. Park and K. Kong, X-ray line signal from 7 keV axino dark matter decay, Phys. Lett. B 733 (2014) 217 [arXiv:1403.1536] [InSPIRE].

[78] L. Lello and D. Boyanovsky, Cosmological Implications of Light Sterile Neutrinos produced after the QCD Phase Transition, Phys. Rev. D 91 (2015) 063502 [arXiv:1411.2690] [INSPIRE].

[79] H. Ishida, K.S. Jeong and F. Takahashi, 7 keV sterile neutrino dark matter from split flavor mechanism, Phys. Lett. B 732 (2014) 196 [arXiv:1402.5837] [INSPIRE].

[80] J.L. Rosner, Three sterile neutrinos in E 6 , Phys. Rev. D 90 (2014) 035005 [arXiv:1404.5198] [INSPIRE].

[81] P.D. Alvarez, J.P. Conlon, F.V. Day, M.C.D. Marsh and M. Rummel, Observational consistency and future predictions for a $3.5 \mathrm{keV}$ ALP to photon line, JCAP 04 (2015) 013 [arXiv: 1410.1867] [INSPIRE].

[82] B. Henning, J. Kehayias, H. Murayama, D. Pinner and T.T. Yanagida, A keV String Axion from High Scale Supersymmetry, Phys. Rev. D 91 (2015) 045036 [arXiv:1408.0286] [INSPIRE].

[83] M. Cicoli, J.P. Conlon, M.C.D. Marsh and M. Rummel, $3.55 \mathrm{keV}$ photon line and its morphology from a $3.55 \mathrm{keV}$ axionlike particle line, Phys. Rev. D 90 (2014) 023540 [arXiv: 1403.2370] [INSPIRE].

[84] A.G. Dias, A.C.B. Machado, C.C. Nishi, A. Ringwald and P. Vaudrevange, The Quest for an Intermediate-Scale Accidental Axion and Further ALPs, JHEP 06 (2014) 037 [arXiv:1403.5760] [INSPIRE].

[85] J.P. Conlon and F.V. Day, $3.55 \mathrm{keV}$ photon lines from axion to photon conversion in the Milky Way and M31, JCAP 11 (2014) 033 [arXiv:1404.7741] [INSPIRE].

[86] K. Hamaguchi, M. Ibe, T.T. Yanagida and N. Yokozaki, Testing the Minimal Direct Gauge Mediation at the LHC, Phys. Rev. D 90 (2014) 015027 [arXiv:1403.1398] [INSPIRE].

[87] C. Kolda and J. Unwin, X-ray lines from R-parity violating decays of ke $V$ sparticles, Phys. Rev. D 90 (2014) 023535 [arXiv: 1403.5580] [INSPIRE].

[88] N.E. Bomark and L. Roszkowski, $3.5 \mathrm{keV}$ x-ray line from decaying gravitino dark matter, Phys. Rev. D 90 (2014) 011701 [arXiv:1403.6503] [InSPIRE].

[89] Z. Kang, P. Ko, T. Li and Y. Liu, Natural X-ray Lines from the Low Scale Supersymmetry Breaking, Phys. Lett. B 742 (2015) 249 [arXiv:1403.7742] [INSPIRE]. 
[90] S.V. Demidov and D.S. Gorbunov, SUSY in the sky or a keV signature of sub-GeV gravitino dark matter, Phys. Rev. D 90 (2014) 035014 [arXiv: 1404.1339] [INSPIRE].

[91] B. Dutta, I. Gogoladze, R. Khalid and Q. Shafi, $3.5 \mathrm{keV}$ X-ray line and R-Parity Conserving Supersymmetry, JHEP 11 (2014) 018 [arXiv:1407.0863] [INSPIRE].

[92] H.M. Lee, C.B. Park and M. Park, Supersymmetric Higgs-portal and X-ray lines, Phys. Lett. B 744 (2015) 218 [arXiv:1501.05479] [INSPIRE].

[93] V. Pavlidou and T.N. Tomaras, Where the world stands still: turnaround as a strong test of $\Lambda C D M$ cosmology, JCAP 09 (2014) 020 [arXiv:1310.1920] [inSPIRE].

[94] J.M. Cline and A.R. Frey, Consistency of dark matter interpretations of the $3.5 \mathrm{keV} \mathrm{x-ray}$ line, Phys. Rev. D 90 (2014) 123537 [arXiv:1410.7766] [INSPIRE].

[95] S. Baek, $3.5 \mathrm{keV} X$-ray Line Signal from Dark Matter Decay in Local U(1) $)_{B-L}$ Extension of Zee-Babu Model, arXiv:1410.1992 [INSPIRE].

[96] A. Falkowski, Y. Hochberg and J.T. Ruderman, Displaced Vertices from X-ray Lines, JHEP 11 (2014) 140 [arXiv:1409.2872] [INSPIRE].

[97] K. Schutz and T.R. Slatyer, Self-Scattering for Dark Matter with an Excited State, JCAP 01 (2015) 021 [arXiv: 1409.2867] [INSPIRE].

[98] K.K. Boddy, J.L. Feng, M. Kaplinghat, Y. Shadmi and T.M.P. Tait, Strongly interacting dark matter: Self-interactions and keV lines, Phys. Rev. D 90 (2014) 095016 [arXiv: 1408.6532] [INSPIRE].

[99] Y. Farzan and A.R. Akbarieh, Decaying Vector Dark Matter as an Explanation for the $3.5 \mathrm{keV}$ Line from Galaxy Clusters, JCAP 11 (2014) 015 [arXiv: 1408. 2950] [INSPIRE].

[100] J.M. Cline and A.R. Frey, Nonabelian dark matter models for 3.5 keV X-rays, JCAP 10 (2014) 013 [arXiv: 1408.0233] [INSPIRE].

[101] C.-W. Chiang and T. Yamada, $3.5 \mathrm{keV} X$-ray line from nearly-degenerate WIMP dark matter decays, JHEP 09 (2014) 006 [arXiv: 1407.0460] [INSPIRE].

[102] C.-Q. Geng, D. Huang and L.-H. Tsai, X-ray Line from the Dark Transition Electric Dipole, JHEP 08 (2014) 086 [arXiv:1406.6481] [INSPIRE].

[103] J.P. Conlon and A.J. Powell, A $3.55 \mathrm{keV}$ line from $D M \rightarrow a \rightarrow \gamma$ : predictions for cool-core and non-cool-core clusters, JCAP 01 (2015) 019 [arXiv:1406.5518] [INSPIRE].

[104] N. Chen, Z. Liu and P. Nath, $3.5 \mathrm{keV}$ galactic emission line as a signal from the hidden sector, Phys. Rev. D 90 (2014) 035009 [arXiv: 1406.0687] [inSPIRE].

[105] K. Nakayama, F. Takahashi and T.T. Yanagida, Extra light fermions in E $E_{6}$-inspired models and the $3.5 \mathrm{keV}$ X-ray line signal, Phys. Lett. B 737 (2014) 311 [arXiv:1405.4670] [INSPIRE].

[106] S. Baek, P. Ko and W.-I. Park, The $3.5 \mathrm{keV}$ X-ray line signature from annihilating and decaying dark matter in Weinberg model, arXiv:1405.3730 [INSPIRE].

[107] H.M. Lee, Magnetic dark matter for the X-ray line at 3.55 keV, Phys. Lett. B 738 (2014) 118 [arXiv:1404.5446] [INSPIRE].

[108] K.S. Babu and R.N. Mohapatra, 7 keV Scalar Dark Matter and the Anomalous Galactic X-ray Spectrum, Phys. Rev. D 89 (2014) 115011 [arXiv:1404.2220] [INSPIRE]. 
[109] E. Dudas, L. Heurtier and Y. Mambrini, Generating X-ray lines from annihilating dark matter, Phys. Rev. D 90 (2014) 035002 [arXiv:1404.1927] [INSPIRE].

[110] F.S. Queiroz and K. Sinha, The Poker Face of the Majoron Dark Matter Model: LUX to keV Line, Phys. Lett. B 735 (2014) 69 [arXiv:1404.1400] [inSPIRE].

[111] K. Nakayama, F. Takahashi and T.T. Yanagida, Anomaly-free flavor models for Nambu-Goldstone bosons and the $3.5 \mathrm{keV}$ X-ray line signal, Phys. Lett. B 734 (2014) 178 [arXiv:1403.7390] [INSPIRE].

[112] R. Allahverdi, B. Dutta and Y. Gao, keV Photon Emission from Light Nonthermal Dark Matter, Phys. Rev. D 89 (2014) 127305 [arXiv: 1403.5717] [INSPIRE].

[113] F. Bezrukov and D. Gorbunov, Relic Gravity Waves and $7 \mathrm{keV}$ Dark Matter from a GeV scale inflaton, Phys. Lett. B 736 (2014) 494 [arXiv:1403.4638] [INSPIRE].

[114] K. Nakayama, F. Takahashi and T.T. Yanagida, The $3.5 \mathrm{keV} X$-ray line signal from decaying moduli with low cutoff scale, Phys. Lett. B 735 (2014) 338 [arXiv:1403.1733] [InSPIRE].

[115] M.T. Frandsen, F. Sannino, I.M. Shoemaker and O. Svendsen, X-ray Lines from Dark Matter: The Good, The Bad and The Unlikely, JCAP 05 (2014) 033 [arXiv:1403.1570] [INSPIRE].

[116] C. El Aisati, T. Hambye and T. Scarnà, Can a millicharged dark matter particle emit an observable gamma-ray line?, JHEP 08 (2014) 133 [arXiv:1403.1280] [INSPIRE].

[117] R. Krall, M. Reece and T. Roxlo, Effective field theory and keV lines from dark matter, JCAP 09 (2014) 007 [arXiv: 1403.1240] [INSPIRE].

[118] D.P. Finkbeiner and N. Weiner, An X-Ray Line from eXciting Dark Matter, arXiv: 1402.6671 [INSPIRE].

[119] K.S. Babu, S. Chakdar and R.N. Mohapatra, Warm Dark Matter in Two Higgs Doublet Models, Phys. Rev. D 91 (2015) 075020 [arXiv:1412.7745] [InSPIRE].

[120] G. Arcadi, L. Covi and F. Dradi, 3.55 keV line in Minimal Decaying Dark Matter scenarios, JCAP 07 (2015) 023 [arXiv: 1412.6351] [INSPIRE].

[121] K. Cheung, W.-C. Huang and Y.-L.S. Tsai, Non-abelian Dark Matter Solutions for Galactic Gamma-ray Excess and Perseus $3.5 \mathrm{keV}$ X-ray Line, JCAP 05 (2015) 053 [arXiv: 1411.2619] [INSPIRE].

[122] H. Baer, K.-Y. Choi, J.E. Kim and L. Roszkowski, Dark matter production in the early Universe: beyond the thermal WIMP paradigm, Phys. Rept. 555 (2014) 1 [arXiv: 1407.0017] [INSPIRE].

[123] A. Biswas, D. Majumdar and P. Roy, Nonthermal two component dark matter model for Fermi-LAT $\gamma$-ray excess and $3.55 \mathrm{keV}$ X-ray line, JHEP 04 (2015) 065 [arXiv:1501.02666] [INSPIRE].

[124] A. Berlin, A. DiFranzo and D. Hooper, $3.55 \mathrm{keV}$ line from exciting dark matter without a hidden sector, Phys. Rev. D 91 (2015) 075018 [arXiv:1501.03496] [INSPIRE]. 